\title{
Formation of heavy quarks in ultrarelativistic heavy-ion collisions
}

\author{
S. M. Schneider and W. Greiner \\ Institut für Theoretische Physik, Johann Wolfgang Goethe-Universität, Postfach 111 932, D-6000 Frankfurt am Main, Germany \\ G. Soff \\ Gesellschaft für Schwerionenforschung (GSI), Planckstrasse 1, Postfach 110552, D-6100 Darmstadt, Germany \\ (Received 18 February 1992)
}

\begin{abstract}
We investigate the production of heavy quarks in continuum and bound states in nuclear collisions. Creation rates for free $b \bar{b}$ and $t \bar{t}$ quark pairs and for bottomonium and toponium in the ground state are computed at energies of the BNL Relativistic Heavy Ion Collider, CERN Large Hadron Collider (LHC), and Superconducting Super Collider. Central and peripheral heavy-ion collisions are discussed. For top-quark creation we assumed a mass range of $90 \leq m_{t} \leq 250 \mathrm{GeV}$. The creation rate for top quarks in peripheral collisions is estimated to be by a factor 40 to 130 smaller compared with corresponding central collisions. For $m_{t}=130 \mathrm{GeV}$ we calculated a creation rate of about 4760 top-quark pairs per day at the $\mathrm{LHC}$ (3.5 TeV/nucleon) for Pb-Pb collisions.
\end{abstract}

PACS number(s): 25.75. $+\mathrm{r}, 14.40 . \mathrm{Gx}, 14.80 . \mathrm{Dq}, 24.85 .+\mathrm{p}$

\section{INTRODUCTION}

The electromagnetic and weak interaction at current energies are excellently described by the standard model. The standard model requires complete fermion families of weak lepton and quark isospin doublets in order to become renormalizable. The heaviest quark that is detected so far is the bottom quark $b$ with a mass of approximately $m_{b} \simeq 4.5 \mathrm{GeV}$. The sixth quark whose existence is postulated to complete the third family is the top quark $t$. Its production in $e^{+} e^{-}$annihilation as well as in hadronic reactions has been extensively discussed and some experimental effort was devoted to the search for clear signals for its generation. In this paper we will focus our attention on the creation of free $b \bar{b}$ and $t \bar{t}$ quark pairs in heavy-ion collisions at ultrarelativistic energies. Additionally we will comment on quarkonium production.

The process $e^{+} e^{-} \rightarrow$ hadrons is well suited for detecting hadronic resonances since electron and positron are elementary particles and the reaction is relatively "clean" compared with hadronic reactions. But, up to the maximum energy, the experiments at the CERN $e^{+} e^{-}$collider LEP resulted in an exclusion of a top quark with a mass lower than $m_{t}<45 \mathrm{GeV} . \bar{p} p$ colliders promise a much higher c.m. energy than present $e^{+} e^{-}$colliders. But, since the elementary constituents of the strong interaction, the quarks and gluons, are confined, a resonance on the parton level will not yield a resonant structure in hadronic cross sections. A search for signals from top quarks in hadronic reactions necessitates a more sophisticated analysis of measured data. Unfortunately, all published data from the UA1, UA2, and Collider Detector at Fermilab (CDF) experiments did not exhibit any evidence for produced top quarks ([1], and references therein). However, these experiments revealed a new lower bound for the top-quark mass:

$$
m_{t} \geq 89 \mathrm{GeV} \text {. }
$$

Several authors published predictions for the top-quark mass. For example, Halzen and Morris concluded from a muon lifetime examination, including the recent precision measurements of $M_{Z}$ and $M_{W}$, that the upper bound for the top-quark mass has to be $m_{t}<260 \mathrm{GeV}$ [2]. Predictions from different investigations agree with this upper bound and favor a mass of $m_{t} \simeq 135-150 \mathrm{GeV}$ [3]. In our calculations, the top-quark mass is varied between $90<m_{t}<250 \mathrm{GeV}$.

The existence of the top quark represents an essential ingredient of the standard model. Moreover, its existence is a prerequisite for explaining the measured charge asymmetry in $B^{0}-\bar{B}^{0}$ mixing [4]. Finally, precise investigations of the $Z^{0}$ line shape resulted in the fundamental discovery that the number of fermion families is limited to three as long as the mass of a fourth neutrino is less than $m_{v}<40 \mathrm{GeV}$ [5]. This is synonymous with the remarkable statement that the top quark will probably be the last elementary fermion to be found.

Regarding the high-mass predictions for the top quark and the Higgs boson, it appears to be unlikely to create these exotic particles at $e^{+} e^{-}$colliders in the near future. Even the boosted LEP 2 collider with its $100 \mathrm{GeV}$ on $100-\mathrm{GeV} e^{+} e^{-}$beam presumably will not provide the possibility to create heavy top quarks. At the Tevatron at Fermilab, the top quark can be detected if it is not heavier than $m_{t} \simeq 120 \mathrm{GeV}$ [6]. For a still heavier top quark, one has to wait until new colliders start to operate. There are planned $e^{+} e^{-}$colliders [e.g., the Japan Linear Collider (JLC) with a c.m. energy of about $500 \mathrm{GeV}$ ] and $p p$ colliders, namely, the Large Hadron Collider (LHC) at CERN and the Superconducting Super Collider (SSC) in Texas, which will accelerate protons up to c.m. energies of 16 and $40 \mathrm{TeV}$, respectively. These two colliders offer the possibility to also collide heavy ions with energies up to $3.5 \mathrm{TeV} /$ nucleon (LHC) and $8 \mathrm{TeV} /$ nucleon (SSC). These ultrarelativistic heavy-ion collisions certainly will produce a large amount of top quarks (and other exotic 
particles such as Higgs bosons or magnetic monopoles and supersymmetric particles, provided they exist in the available mass range). A heavy-ion collision has one important creation channel which is insignificant for hadronic collisions: the peripheral heavy-ion collision with a large impact parameter at which parton-parton reaction can be completely excluded and at which the hadronic background is drastically reduced $(\gamma \gamma$ fusion and $\gamma g$ fusion are the elementary subprocesses). In the next sections we discuss the cross sections for forming heavy quarks in central as well as in peripheral heavy-ion collisions and the detectability of the produced quarks.

\section{NUCLEAR COLLISIONS}

We discuss two different configurations for ultrarelativistic nuclear collisions: the central collision and the peripheral collision. In central nuclear collisions we are dealing with more than 200 nucleons colliding with more than 200 other nucleons at c.m. energies of several $\mathrm{TeV}$ per nucleon, i.e., at a total c.m. energy of more than 1000 $\mathrm{TeV}$ (a Pb-Pb collision at $3.5 \mathrm{TeV} /$ nucleon corresponds to a total c.m. energy of $1456 \mathrm{TeV}$ ). The maximum energy of $8 \mathrm{TeV} /$ nucleon at the SSC would be equivalent to a fixed targeted energy of $E_{\text {lab }} \simeq 1.28 \times 10^{8} \mathrm{GeV} /$ nucleon. It is obvious that such an enormous energy will generate a firework of created particles.

At present energies, which are available at the CERN heavy-ion accelerator, the data still exhibit stopping of the impinging nuclei. But at an energy which is increased by about 6 orders of magnitude, one expects a more transparent behavior of the nuclei. The nucleonic constitutents of the nuclei should retain a high fraction of their initial four-momenta. This postulated behavior is the basis of the "simple incoherence model" we are using in order to calculate the total cross section for nuclear collisions. The underlying assumptions of this model are (see also [9]) (1) a dominant transparency of extremely relativistic moving nuclei in a collision, (2) the neglect of nucleon-nucleon interactions inside a single nucleus (the nuclei are treated as clusters of noninteracting nucleons; the transverse momentum is uncared for), and (3) the assumption that the nuclear cross sections are obtained by an incoherent sum over binary nucleon-nucleon collisions. This means that we are reducing the complex problem of an ultrarelativistic heavy-ion collision to a consideration of the binary nucleon collisions. Obvious$\mathrm{ly}$, this is a first approximation, standing for a more sophisticated examination in future calculations.

The program we have to manage is as follows: (a) determine the number of binary collisions occurring at a fixed impact parameter $b$, (b) compute the nucleonic cross section for producing the considerable final state, and (c) calculate the nuclear cross section.

The problem of counting the number of binary collisions at a fixed impact parameter $b$ is simplified to a geometrical analysis: at first we utilize the following parametrization of the total nucleon-nucleon cross section [7]:

$$
\sigma_{\text {tot }}(S)=\left[38.3+0.43 \ln ^{2}\left(\frac{S}{100 \mathrm{GeV}^{2}}\right)\right] \mathrm{mb},
$$

where $S$ is the squared center-of-momentum energy of the colliding nucleons. We do not distinguish between $p p$, $p n$, or $n n$ collisions because of the small contribution of the quark annihilation process to the total nucleonic cross section; the cross section is predominantly determined by gluons. The second step is to center a circle around each nucleon of nucleus 1 perpendicular to the linear assumed trajectory, where the area of the circle is given by the total cross section (1). The third and last step is to project nucleus 1 on nucleus 2 with given impact parameter $b$. The number of binary collisions which will occur during the collision is taken as the number of penetrations of nucleons from nucleus 2 through the circles centered round the nucleons of nucleus 1. After computation of $n(b)$, the number of binary collisions as a function of the impact parameter, it is straightforward to calculate the total nuclear cross section. For spherical nuclei the total cross section reads

$\sigma_{A A}(X ; S)=\frac{2 \pi}{\sigma_{\text {tot }}(S)} \int_{0}^{2 \widetilde{R}} d b n(b) b \sigma(n n \rightarrow X ; S)$,

where $X$ signifies the final state we are considering and $\widetilde{R}$ is the minimum radial distance between the centers of the nuclei at which no binary collisions will occur. $\sigma(n n \rightarrow X ; S)$ is introduced to denote the total hadronic cross section. To initialize the nuclei and to compute $n(b)$, we used a simplified code of the relativistic quantum molecular dynamics (RQMD) model [8]. Two results for $n(b)$ are plotted as a function of $b$ in Fig. 1. The number of binary collisions at $b=0$ is compatible with the number calculated according to $n(b=0) \simeq A^{4 / 3}$.

Before discussing the peripheral collision we have to define what is meant with the phrase "peripheral." In this paper peripheral collisions are collisions with large

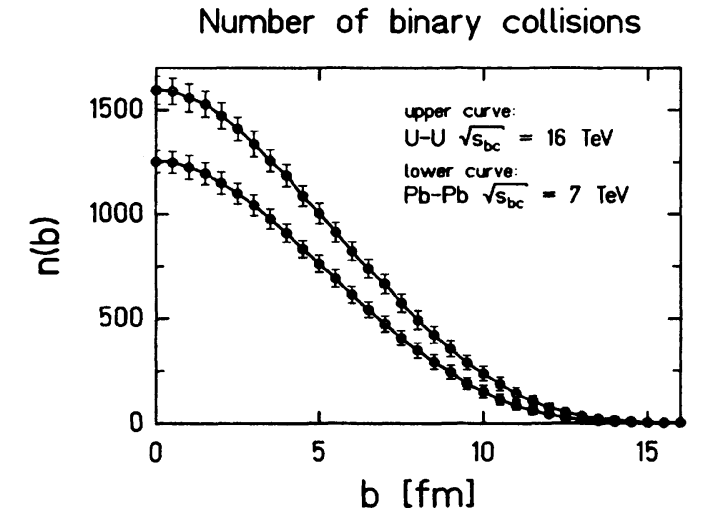

FIG. 1. Number of binary collisions in a central heavy-ion collision at fixed impact parameter plotted as a function of the impact parameter. $n(b)$ is computed within the simple incoherence model, employing a simplified RQMD model code [8]. The upper line corresponds to the SSC energy and U-U collisions while the lower line is calculated for LHC energy and $\mathrm{Pb}-\mathrm{Pb}$ collisions. 
impact parameters at which no direct hard parton-parton reactions can occur, e.g., in collisions with an impact parameter larger than twice the nuclear radius no hard parton-parton collision will take place. Though direct parton-parton reactions are negligible, indirect partonparton reactions, such as diffractive processes, are still possible. But these more indirect reactions are not taken into account in our present calculations.

Why do we examine the peripheral collision, which is usually ignored in hadronic collisions? The answer is that the strength of the electromagnetic field of a relativistic moving charge depends quadratically on the charge number $Z$; e.g., the electromagnetic field of a lead nucleus is 6724 times stronger than the field of a proton. Furthermore, the electromagnetic field of an ultrarelativistic moving charge is almost transversal. Thus, the field of a high-energy nucleus is describable as a swarm of real photons [10]. This description is traditionally known as the method of equivalent photons. The expression for the photon distribution of an arbitrary charge distribution reads [11]

$$
n(\omega)=\frac{2 \alpha}{\pi} Z^{2} \frac{1}{\omega} \int_{\gamma / \omega}^{\infty} d k \frac{k^{2}-(\omega / \gamma)^{2}}{k^{3}} F^{2}(k),
$$

where $F(k)$ denotes the elastic form factor of the nucleus, i.e., the Fourier transform of the charge distribution, and $\gamma=E / m$ is the Lorentz contraction factor. We are using the photon distribution of a homogeneously charged sphere [12]:

$$
\begin{aligned}
f(x)= & \frac{3}{16 x^{6}}+\frac{3}{8 x^{4}}-\cos (2 x)\left[\frac{3}{16 x^{6}}+\frac{7}{40 x^{2}}+\frac{1}{20}\right] \\
& -\sin (2 x)\left[\frac{3}{8 x^{5}}+\frac{1}{10 x^{3}}-\frac{9}{20 x}-\frac{x}{10}\right] \\
& -\left[1+\frac{x^{2}}{5}\right] \operatorname{Ci}(2 x)
\end{aligned}
$$

with the integral-cosine function

$$
\operatorname{Ci}(z)=-\int_{z}^{\infty} d \tau \frac{\cos \tau}{\tau}
$$

to be inserted into

$$
n(\omega)=\frac{2 \alpha}{\pi} Z^{2} \frac{1}{\omega} f\left(\omega / \omega_{\max }\right) .
$$

A rough estimate of the maximum photon frequency $\omega_{\max }=\gamma / R_{\text {nuc }} \quad$ yields $\omega_{\max }(\mathrm{LHC}) \simeq 100 \mathrm{GeV}$ and $\omega_{\max }(\mathrm{SSC}) \simeq 230 \mathrm{GeV}$, where we inserted $\gamma_{\mathrm{LHC}}=3500$, $\gamma_{\text {ssC }}=8000$, and $R_{\text {nuc }}=7 \mathrm{fm}$. Because of the relatively small maximum photon frequency at the LHC compared with the top-quark mass, photon-photon fusion is only of minor interest. More important is the photon-gluon fusion, where the gluons will carry the required energy and the strong-coupling constant will amplify the reaction rate. The computation of particle creation cross sections is presented in the next section.

\section{ELEMENTARY CROSS SECTIONS}

In the previous section we described the relationship of nuclear cross sections with nucleon-nucleon scattering. The computation of hadronic collisions at relativistic energies is usually performed in the framework of the parton model, i.e., a nucleon is described as a swarm of noninteracting particles, called partons. These partons are the valence quarks, the gluons, and the sea quarks. The dynamical behavior of the parton distributions is determined by solutions of the coupled Altarelli-Parisi equations for the different parton types [13]. The Altarelli-Parisi equations include first-order radiative corrections that are not taken into account in the naive parton model, i.e., quantum field-theoretical corrections are ignored in the naive parton approach. For realistic calculations of very heavy-particle creation, the radiative corrections are indispensable. The solution of the Altarelli-Parisi equations incorporating related experimental data at different c.m. energies as input will yield a parametrization of the parton distributions. The parton distributions depend on $x$ and $Q^{2}$, where $x$ is the fraction of the four-momentum of the mother nucleon carried by the parton and $Q^{2}$ stands for the squared fourmomentum transfer. This analysis was accomplished by several authors; we adopt the parametrization of Duke and Owens [14]. Since we are not testing the small- $x$ region $\left(x<10^{-2}\right)$ when producing heavy quarks, this parametrization might result in cross sections that do not deviate from those obtained with different parametrizations. Employing the parton model, we are reaching a deeper sublevel of approximation for the description of a nuclear collision. The first step was to describe the nuclei as a cluster of independent nucleons. Within the parton model the nucleons are clouds of noninteracting constituents during the collision. The hadronic cross section is now reduced to an integral over the parton distributions of the impinging nucleons and the associated elementary cross section:

$$
\begin{aligned}
\sigma(n n \rightarrow X ; S)=\sum_{i, j} \int & d x_{1} d x_{2} f_{i}\left(x_{1}, Q^{2}\right) f_{j}\left(x_{2}, Q^{2}\right) \\
& \times \sigma_{i j}(X ; \hat{s}) \Theta\left(\widehat{s}-m_{X}^{2}\right) .
\end{aligned}
$$

Here $S$ denotes the squared invariant energy of the colliding nucleons, $Q^{2}$ is the squared four-momentum transfer, and $i$ and $j$ distinguish between the different kinds of partons. Furthermore, $x_{1}$ and $x_{2}$ are the four-momentum fractions of the mother nucleons, carried by the partons and $\hat{s}$ is the squared invariant energy of the parton subprocess, depending on $x_{1}$ and $x_{2}$. The $\Theta$ function ensures that the invariant parton energy $\sqrt{\hat{s}}$ is at least the mass $m_{X}$ of the created particles. In our calculations the parton distributions $f_{i}\left(x, Q^{2}\right)$ are evolved at $Q^{2}=\hat{s}$ for the quark-antiquark annihilation process and at $Q^{2}=\frac{1}{4} m_{X}^{2}$ for fusion processes. Assuming a vanishing effective gluon mass, the functional dependence between $\widehat{s}$ and $S$ is simply

$$
\hat{s}=x_{1} x_{2} S .
$$

Figure 2 illustrates a hadronic collision within the parton model. 


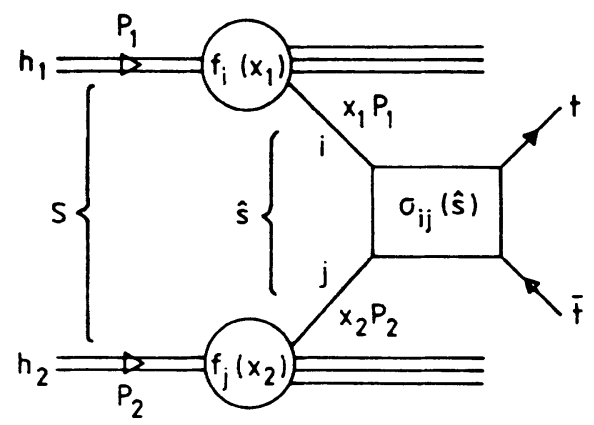

FIG. 2. Graphical illustration of hadronic collisions in the parton point of view. The hadrons $h_{1}$ and $h_{2}$ with the fourmomenta $P_{1}$ and $P_{2}$ collide with the invariant energy $\sqrt{S}$. The $f_{i}(x)$ are the parton distribution functions for the hadrons, with $i$ distinguishing between the different parton types. $\sqrt{\hat{s}}$ denotes the invariant energy of the parton-parton reaction.

As central object of our investigations we are dealing with heavy-ion collision scenarios. In central collisions the gluon fusion and the various quark-antiquark annihilation subprocesses are contributing most to the hadronic cross section. In peripheral collisions the photon-photon and the photon-gluon fusion subprocesses take this place due to the vanishing hard parton-parton processes. Since we are calculating the cross sections up to second-order perturbation theory only, it is important whether or not the considered final state is generated in a specified process. For example, it is obvious that, in lowest-order perturbation theory, the quark-antiquark annihilation process via a virtual gluon will not contribute to the creation of quarkonia because color conservation rules out this process (the gluon is a color octet and physical states are colorless).

In the following we indicate diverse elementary cross sections that are relevant for our calculations. All cross sections are computed in second-order perturbation theory $\left(\alpha_{s}^{2}, \alpha_{s} \alpha_{\mathrm{em}}, \alpha_{\mathrm{em}}^{2}\right)$. A more detailed derivation of these cross sections is presented in Appendix A. Most important for the central collisions up to Tevatron energies is the quark-antiquark annihilation process into a free heavy-quark pair:

$$
\begin{aligned}
\sigma_{q \bar{q}}(Q \bar{Q} ; \hat{s})= & \frac{2}{9} \frac{4 \pi \alpha_{s}^{2}\left(m_{Q}^{2}\right)}{3 \hat{s}}\left[\frac{\hat{s}-4 m_{Q}^{2}}{\hat{s}-4 m_{q}^{2}}\right)^{1 / 2} \\
& \times\left[1+\frac{2 m_{Q}^{2}}{\hat{s}}\right]\left(1+\frac{2 m_{q}^{2}}{\hat{s}}\right),
\end{aligned}
$$

where $m_{q}$ and $m_{Q}$ are the masses of the ingoing and outgoing quarks, respectively. $\alpha_{s}\left(m_{Q}^{2}\right)$ denotes the running coupling constant of the strong interaction, evaluated at the squared mass of the formed quark. Neglecting the light-quark masses $m_{q}$, Eq. (9) leads to the actually applied formula

$$
\sigma_{q \bar{q}}(Q \bar{Q} ; \widehat{s}) \simeq \frac{2}{9} \frac{4 \pi \alpha_{s}^{2}\left(m_{Q}^{2}\right)}{3 \widehat{s}^{2}}\left(\widehat{s}+2 m_{Q}^{2}\right) \Delta_{T}
$$

with the threshold function

$$
\Delta_{T}=\left(1-4 m_{Q}^{2} / \hat{s}\right)^{1 / 2} \text {. }
$$

The evaluation of the gluon fusion process, which becomes most important for LHC and SSC energies, yields

$$
\begin{aligned}
\sigma_{g g}(Q \bar{Q} ; \hat{s})=\frac{\pi \alpha_{s}^{2}\left(m_{Q}^{2}\right)}{3 \hat{s}}[ & \frac{1}{4}\left[-7-\frac{31 m_{Q}^{2}}{\hat{s}}\right) \Delta_{T} \\
+ & {\left[1+\frac{4 m_{Q}^{2}}{\widehat{s}}+\frac{m_{Q}^{4}}{\widehat{s}^{2}}\right) } \\
& \left.\times \ln \left\{\frac{1+\Delta_{T}}{1-\Delta_{T}}\right\}\right] .
\end{aligned}
$$

Considering the creation of $1{ }^{1} S_{0}$ quarkonia leads to a different gluon fusion process:

$$
\sigma_{g g}\left({ }^{1} S_{0} ; \hat{s}\right)=\frac{\pi^{2} \alpha_{s}^{2}}{3 M_{\eta}^{3}}\left|R_{S}(0)\right|^{2} \delta\left(\hat{s}-M_{\eta}^{2}\right),
$$

with $M_{\eta}$ the mass of the $1{ }^{1} S_{0}$ quarkonium and $R_{S}(0)$ the radial wave function at the origin.

In peripheral collisions pure parton processes are forbidden. We have to treat processes containing at least one photon in the fusion channel (as long as we are neglecting the hadronic structure of the photon field). This selection rule implies two combinations. The only process in peripheral collisions that involves a parton as an ingoing particle is the photon-gluon fusion process into a heavy-quark-antiquark pair [15]. The similarity of the parton picture and the above-mentioned equivalent photons leads to the following integral over the elementary cross section, the photon distribution, and the effective gluon distribution of the nucleus $g_{\text {eff }}^{A}\left(x, Q^{2}\right)$, which includes nuclear shadowing effects of the surface nucleons:

$$
\begin{aligned}
\sigma(A A \rightarrow t \bar{t})=\int & d \omega d x n(\omega) g_{\mathrm{eff}}^{A}\left(x, Q^{2}\right) \\
& \times \sigma(\gamma g \rightarrow t \bar{t}) \Theta\left(\widehat{s}-4 m_{t}^{2}\right) .
\end{aligned}
$$

Experimental data support a simple functional dependence of the nuclear gluon distribution on the mass number [16]:

$$
g_{\mathrm{eff}}^{A}\left(x, Q^{2}\right)=A_{\mathrm{eff}} g\left(x, Q^{2}\right) \simeq A^{0.9} g\left(x, Q^{2}\right) .
$$

The calculation of the elementary photon-gluon fusion cross section results in the expression

$$
\begin{aligned}
\sigma_{\gamma g}(Q \bar{Q} ; \hat{s})=\frac{4 \pi \alpha \alpha_{s} Q_{Q}^{2}}{2 \widehat{s}}[ & -\left(1+\frac{4 m_{Q}^{2}}{\hat{s}}\right) \Delta_{T} \\
+ & {\left[1+\frac{4 m_{Q}^{2}}{\hat{s}}-\frac{8 m_{Q}^{4}}{\hat{s}^{2}}\right] } \\
& \left.\times \ln \left\{\frac{1+\Delta_{T}}{1-\Delta_{T}}\right\}\right],
\end{aligned}
$$

where $Q_{Q}$ is the fractional charge of the produced quark in units of the elementary charge $e$. Additionally we consider the photon-photon fusion process 


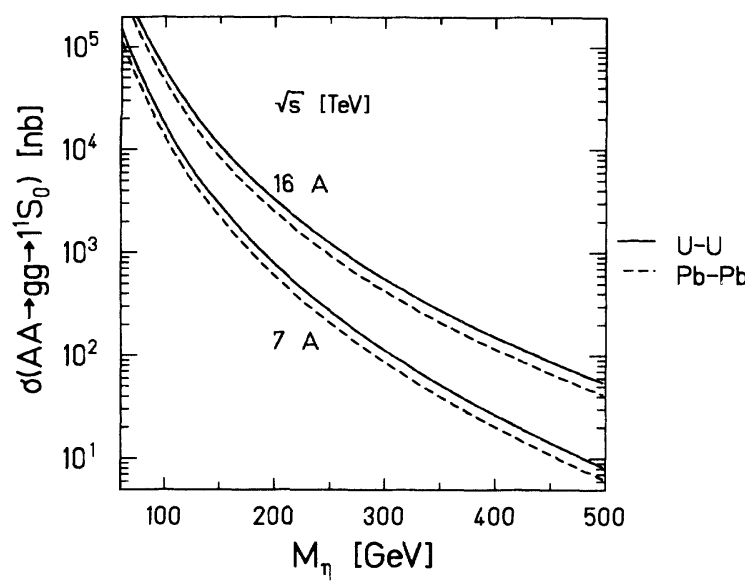

FIG. 3. Total cross section for $1{ }^{1} S_{0}$-toponium creation in central heavy-ion collisions, displayed as a function of the toponium mass $\boldsymbol{M}_{\eta}$. The upper two lines are the results for U-U (bold line) and $\mathrm{Pb}-\mathrm{Pb}$ (dashed line) collisions at the maximum SSC energy ( $8 \mathrm{TeV} /$ nucleon). The lower two lines are the same for the maximum LHC energy (3.5 TeV/nucleon).

$$
\begin{aligned}
\sigma_{\gamma \gamma}(Q \bar{Q} ; \hat{s})=\frac{4 \pi \alpha^{2} Q_{Q}^{4}}{\hat{s}}[ & -\left(1+\frac{4 m_{Q}^{2}}{\hat{s}}\right) \Delta_{T} \\
+ & 2\left(1+\frac{4 m_{Q}^{2}}{\hat{s}}-\frac{8 m_{Q}^{4}}{\widehat{s}^{2}}\right) \\
& \left.\times \ln \left\{\frac{1+\Delta_{T}}{1-\Delta_{T}}\right\}\right] .
\end{aligned}
$$

After deriving all elementary cross sections, we can start to determine total cross sections for heavy-quark formation in nuclear collisions by performing the indicated integrations. Figure 3 displays the total cross section for ${ }^{1} \mathrm{~S}_{0}$-toponium creation in $\mathrm{U}-\mathrm{U}$ and $\mathrm{Pb}-\mathrm{Pb}$ central collisions at $\mathrm{LHC}(3.5 \mathrm{TeV} /$ nucleon $)$ and $\mathrm{SSC}(8.0$

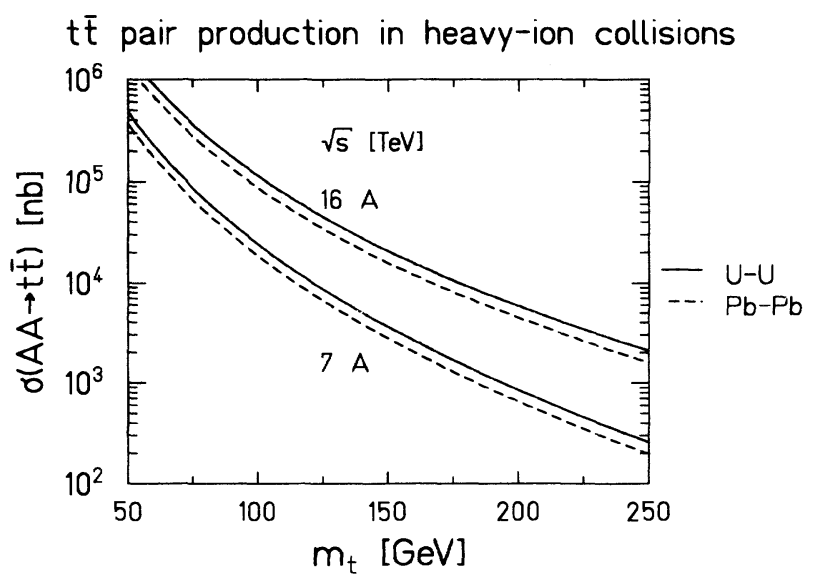

FIG. 4. Total cross section for top-quark pair creation at LHC and SSC energies in central collisions as a function of the top-quark mass $m_{t}$. The solid lines indicate U-U collisions and the dashed lines $\mathrm{Pb}-\mathrm{Pb}$ collisions, respectively. $t \overline{\mathrm{t}}$ creation in peripheral collisions

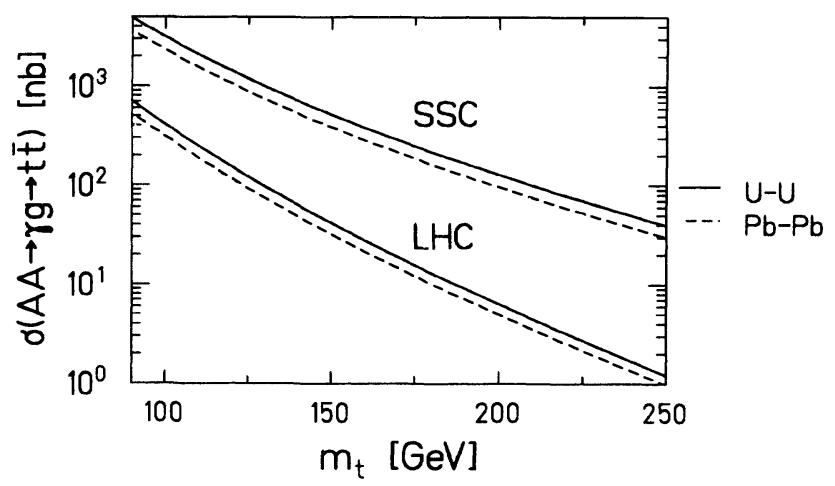

FIG. 5. Total cross section for top-quark pair creation at LHC and SSC energies in peripheral collisions as a function of the top-quark mass. The denotation of the lines is the same as in Fig. 4.

$\mathrm{TeV} /$ nucleon) energies. For the same collision configurations, the free top-quark pair creation is plotted in Fig. 4 as a function of the top-quark mass $m_{t}$. For peripheral collisions, where quarkonium production is suppressed, the total cross section for top-quark pair creation is displayed in Fig. 5.

Since the curves in Fig. 3 imply that the toponia are instantaneously created without taking into account the formation time, we call such states "bachelor" toponia. This indicates that the top quarks still have to get "married" before they become a physical meson state (see also next section).

The curves in Fig. 5 represent results which are integrated over all impact parameters. To obtain a realistic result for pure peripheral collisions one has to introduce a suppression factor $K$ that accounts for contributions from central collisions. It was demonstrated that the suppression factor has to be about $K \simeq \frac{1}{2}$ [17].

A typical value for the cross section for top-quark pair creation in central collisions is $\sigma$ (central) $=5 \mu \mathrm{b}$ for the favored top-quark mass region and LHC energy (3.5 $\mathrm{TeV} /$ nucleon). The toponium creation cross section is lower by about a factor 40, assuming the same configuration. The top-quark pair creation cross section in peripheral collisions is roughly 2 orders of magnitude lower, but the reduction factor varies with the collision

TABLE I. Cross sections and creation rates for $b \bar{b}$ pairs and for bottomonium in the $1{ }^{1} S_{0}\left(\eta_{t}\right)$ and $2{ }^{1} S_{0}\left(\eta_{t}^{\prime}\right)$ states at RHIC energy (100 GeV/nucleon). A bottom-quark mass of $m_{b}=4.5$ $\mathrm{GeV}$ and a luminosity of $\mathcal{L}=10^{26} \mathrm{~cm}^{-2} \mathrm{~s}^{-1}$ is assumed.

\begin{tabular}{llrrr}
\hline \hline & & $b \bar{b}$ & \multicolumn{1}{c}{$\eta_{b}$} & $\eta_{b}^{\prime}$ \\
\hline Central collision & $\sigma(\mu \mathrm{b})$ & 3817 & 1368 & 501 \\
& Rate (per h) & 1374 & 492 & 180 \\
& & & & \\
Peripheral collision & $\sigma(\mu \mathrm{b})$ & 120 & & \\
& Rate (per h) & 43 & & \\
\hline \hline
\end{tabular}


TABLE II. Cross sections and creation rates for top-antitop-quark pairs and toponia in the ground state in central and peripheral collisions at LHC and SSC energies using Pb or U nuclei as collision partners.

\begin{tabular}{|c|c|c|c|c|c|c|}
\hline & & & \multicolumn{2}{|c|}{$m_{t}=130 \mathrm{GeV}$} & \multicolumn{2}{|c|}{$m_{t}=200 \mathrm{GeV}$} \\
\hline & & & $t \bar{t}$ & $\eta_{t}$ & $t \bar{t}$ & $\eta_{t}$ \\
\hline \multirow[t]{2}{*}{ LHC } & Central & $\sigma(\mathrm{nb})$ & 5507 & 178 & 651 & 21 \\
\hline & & Rate (per day) & 4758 & 158 & 562 & 18 \\
\hline \multirow[t]{2}{*}{$\mathrm{Pb}-\mathrm{Pb}$} & Peripheral & $\sigma(\mathrm{nb})$ & 74 & & 5 & \\
\hline & & Rate (per day) & 64 & & $\simeq 4$ & \\
\hline \multirow[t]{2}{*}{ LHC } & Central & $\sigma(\mathrm{nb})$ & 7187 & 233 & 849 & 27 \\
\hline & & Rate (per day) & 6209 & 201 & 733 & 23 \\
\hline \multirow{2}{*}{ U-U } & Peripheral & $\sigma(\mathrm{nb})$ & 97 & & 6.4 & \\
\hline & & Rate (per day) & 84 & & $\simeq 5$ & \\
\hline \multirow[t]{2}{*}{ SSC } & Central & $\sigma(\mathrm{nb})$ & 29234 & 819 & 4453 & 118 \\
\hline & & Rate (per day) & 25258 & 708 & 3847 & 102 \\
\hline \multirow[t]{2}{*}{$\mathrm{Pb}-\mathrm{Pb}$} & Peripheral & $\sigma(\mathrm{nb})$ & 741 & & 96 & \\
\hline & & Rate (per day) & 640 & & 83 & \\
\hline \multirow[t]{2}{*}{ SSC } & Central & $\sigma(\mathrm{nb})$ & 38277 & 1072 & 5831 & 155 \\
\hline & & Rate (per day) & 33458 & 926 & 5038 & 134 \\
\hline \multirow[t]{2}{*}{ U-U } & Peripheral & $\sigma(\mathrm{nb})$ & 998 & & 128 & \\
\hline & & Rate (per day) & 862 & & 111 & \\
\hline
\end{tabular}

configuration. From Table II we learn that the reduction factor between central and peripheral creation $r=\sigma($ central $) / \sigma$ (peripheral) depends on the top-quark mass $m_{t}$ and on the collision energy but not on the mass number $A$ (or on $Z$ ), e.g., one computes $r$ (LHC, $m_{t}=200$ $\mathrm{GeV}) \simeq 130$ and $r\left(\mathrm{SSC}, m_{t}=130 \mathrm{GeV}\right) \simeq 39$.

In addition to the interesting top-quark creation cross sections, we computed bottom-quark and ${ }^{1} S_{0}$ bottomonium creations at the energy of the BNL Relativistic Heavy Ion Collider (RHIC) (100 GeV/nucleon). Table I contains cross sections and creation rates for $\mathrm{Au}$ $\mathrm{Au}$ collisions with an assumed bottom-quark mass of $m_{b}=4.5 \mathrm{GeV}$ and a RHIC luminosity of $\mathcal{L}=10^{26}$ $\mathrm{cm}^{-2} \mathrm{~s}^{-1}$. At RHIC energy, we also employed the simple incoherence model, though heavy-ion collision models show no evident transparent behavior at this energy.

In Table II we present values for the total cross sections of a top-quark pair and $\eta_{t}$-toponium generation. For the LHC and the SSC, a luminosity of $\mathcal{L}=10^{28}$ $\mathrm{cm}^{-2} \mathrm{~s}^{-1}$ is assumed.

\section{DECAY AND DETECTABILITY}

The predicted massive top quark is a very short-lived particle. This follows from its large decay width. The only decay channel of the free top quark is the weak decay into a bottom quark and a $W$ boson. We neglect the mixing of the bottom quark according to the CabibboKobayashi-Maskawa mass matrix where $V_{t b}=1$ is a good approximation. Since the mass of the top quark is larger than the sum of the $W$-boson mass and the bottom-quark mass $m_{t}>m_{W}+m_{b}$, the $W$ boson is assumed to be real. Working out the elementary decay process one obtains (see Appendix B for some calculation steps)

$$
\begin{aligned}
\Gamma\left(t \rightarrow b W^{+}\right)= & \frac{m_{t}^{3} \alpha\left(m_{W}^{2}\right)}{16 m_{W}^{2} \sin ^{2} \vartheta_{W}} \lambda^{1 / 2} \\
& \times\left[\frac{m_{W}^{2}}{m_{t}^{2}}\left[1+\frac{m_{b}^{2}}{m_{t}^{2}}\right]+\left[1-\frac{m_{b}^{2}}{m_{t}^{2}}\right]^{2}\right. \\
& \left.-2 \frac{m_{W}^{4}}{m_{t}^{4}}\right]
\end{aligned}
$$

with the abbreviation

$\lambda\left[1, \frac{m_{b}^{2}}{m_{t}^{2}}, \frac{m_{W}^{2}}{m_{t}^{2}}\right] \equiv \lambda(x, y, z)=(x-y-z)^{2}-4 y z$.

Neglecting $m_{b}^{2} / m_{t}^{2} \ll 1$, Eq. (18) is transformed into

$$
\begin{aligned}
\Gamma\left(t \rightarrow b W^{+}\right) & \simeq \frac{m_{t}^{3} \alpha\left(m_{W}^{2}\right)}{16 m_{W}^{2} \sin ^{2} \vartheta_{W}}\left(1-\frac{m_{W}^{2}}{m_{t}^{2}}\right)^{2}\left(1+\frac{2 m_{W}^{2}}{m_{t}^{2}}\right) \\
& \simeq 581 \mathrm{MeV},
\end{aligned}
$$

where we inserted $m_{W}=80.6 \mathrm{GeV}, \sin ^{2} \theta_{W}=0.2259$, $m_{t}=135 \mathrm{GeV}$, and $\alpha\left(m_{W}^{2}\right)=\frac{1}{128}$. This implies a lifetime of about $\tau_{t} \simeq 0.34 \mathrm{fm} / c$ or less for a heavier top quark.

Since the binding energy of the toponium is relatively small compared with the mass of the top quark, one can utilize the spectator model, in which the two quark components of the quarkonium are treated as independent from each other. This yields a toponium decay width that is twice the decay width of the free top quark. Because of this short lifetime of the top quark, it is necessary to take into account the formation time of the bound state which is assumed to be [18]

$$
t_{\text {form }} \simeq(1 \mathrm{GeV})^{-1}\left[\frac{100 \mathrm{GeV}}{m_{t}}\right] \text {, }
$$


i.e., the top-quark pair does not act instantaneously as a physical bound state. Hence, the effective number of created toponia is reduced. In the favored top-quark mass region, this factor is about $n_{\mathrm{eff}}=\frac{1}{2} n$, where $n$ denotes the number of created "bachelor" toponia. However, this reduction factor could be canceled by a supplementary potential due to a Higgs-boson exchange [19]. To estimate the rate of resonant toponia decays one has to compare the resonant decay channels with the weak decay-or so-called single quark decay (SQD). The weak decay does not appear as a resonant decay experimentally since not all decay products are measured coincidently; hence, the weak decay products are not associated with a peak structure. The evaluation of the dominant resonant decay channel, the decay into two gluons, yields [20]

$$
\Gamma\left(1{ }^{1} S_{0} \rightarrow g g\right)=\frac{8}{3} \frac{\alpha_{s}^{2}\left(m_{t}^{2}\right)}{M_{\eta t}^{2}}\left|R_{S}(0)\right|^{2} .
$$

For the relevant top-quark mass region this is only a small fraction of the nonresonant SQD-decay width $\left[\Gamma(\mathrm{SQD}) / \Gamma(g g) \simeq 330\right.$ for $\left.m_{t} \simeq 135 \mathrm{GeV}\right]$. We have to conclude that searching for the resonant decay products of created toponia in central or peripheral heavy-ion collisions at future colliders or even in $p p$ collision appears to be difficult because this is equivalent to a total resonant decay rate of about 1 event per 2-day beam time.

Thus, we focus our attention on continuum states of the top quark. What are the products of a top-quark pair decay? Clearly there will be two hadronic jets with relatively high transverse momenta $p_{1}$ descending from the bottom quarks. The $W$ boson will decay into a leptonneutrino pair (e.g., $W^{-} \rightarrow e^{-}+v_{e}$ ) or a quark-antiquark pair (e.g., $W^{-} \rightarrow d^{\prime}+\bar{u}$, with $d^{\prime}$ the mixed weak eigenstate). There are three leptonic decay modes of the $W$ boson and six quark-antiquark decay modes which all exhibit roughly a partial decay width that is $\Gamma_{\mathrm{par}} \simeq \frac{1}{9} \Gamma_{\mathrm{tot}}$. The interesting decays of the two $W$ bosons are the purely leptonic decays that amount to above $\frac{1}{9}$ of all possible decay modes. The restriction to a special combination (e.g., $e^{+} \mu^{-}$) suppresses the total creation rate of topquark pairs to a fraction of $\frac{1}{81}$.

As indicated before, there are two important collision scenarios to create the top quark. In central collisions the creation rate of top quarks is relatively high. This sounds promising but experimentally it will be difficult to resolve all generated reaction products. It was shown by Baer et al. [21] that there are two major background processes to the top-quark pair decay: the production of a $W$-boson pair with two additional jets and the $Z^{0}$ production with additional jets. Both processes will result in two different charged leptons and jets. Other background processes are negligible as long as the experimental cuts in the transverse momentum are sufficiently restricted.

The creation rate of top quarks in the peripheral collision is only a minor fraction of the total creation ratebut it is still in the same order of magnitude as the expected creation rate at the Tevatron. This loss of creation rate is the price one has to pay for the drastically suppressed hadronic background. Not only the multiplicity of produced particles is reduced; moreover, the major background processes occurring in the central collision are minimized in the photon-gluon fusion since color conservation requires a radiated gluon in the outgoing channel. It follows that the experimental cuts need not be so restrictive as in the central collision case. The remaining problem is the clear identification of peripheral collisions which is still an interesting field of theoretical examinations.

\section{CONCLUSIONS}

Recapitulating the facts, we conclude that it appears to be difficult to look for signals from toponium in hadronic collisions; even at the SSC, resonant toponium decay will be a relatively rare process. However, free quark pair production could be successful.

In this respect we have to emphasize that we are simply assuming that free top quarks exist on the parton level. This is not trivial at all since the coupling constant between the Higgs boson and the top quark is of order $g_{H t} \simeq 1$.

Central collisions produce top-quark pairs with a high rate of many hundred pairs per hour but equivalently thousands of other hadronic products will accompany the top quarks. It is not obvious whether the decay products of the top-quark pair can be isolated unambiguously from the enormous hadronic background.

Comparing the creation rate for top quarks in central and peripheral collisions at LHC and SSC energies as presented in Table II, the peripheral collisions appear to be of negligible importance compared with the high rates in central collisions (between a few hundred and several thousand created top-quark pairs per day at LHC and up to some 10000 created top-quark pairs per day at SSC). But recalling the fact that the creation rate for top quarks at Tevatron is not higher than in future peripheral collisions at LHC, peripheral collisions still remain an attractive alternative to central collisions.

For strict statements concerning the peripheral collision scenario, one has to await a quantitative analysis of the transverse cross sections for top-quark pair creation and the related background processes. In addition, one has to allow for diffractive creation channels. But we assume that our discussion has pointed out the dominant features.

\section{APPENDIX A}

The interaction of quarks and gluons is characterized by color-SU(3) symmetry. Figures 6-8 display the Feynman graphs for the quark-antiquark annihilation (Fig. 6), the gluon-gluon fusion (Fig. 7), and the photon-gluon fusion (Fig. 8) in second-order perturbation theory. The photon-gluon fusion is not a pure QCD graph but includes the electroweak interaction of the quarks. These processes have been calculated by several authors (see, e.g., [22] for $g g$ fusion and [23] for $\gamma g$ fusion). For the sake of completeness we present the calculation, especially for the $g g$-fusion process, in some detail.

The $S$-matrix element between a quantum-mechanical initial state $|i\rangle$ and a final state $|f\rangle$ is defined through 


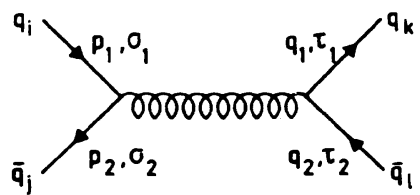

FIG. 6. Second-order perturbation theory Feynman diagram for the quark-antiquark annihilation. $\sigma_{i}$ and $\tau_{i}$ are the spins of the ingoing and outgoing particles.

a)

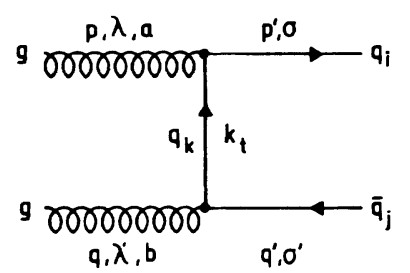

b)

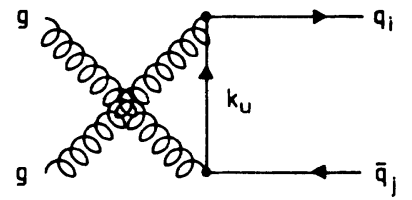

c)

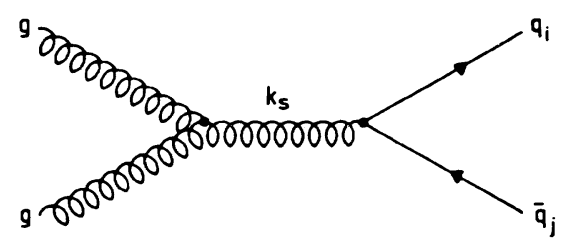

FIG. 7. The three coherent contributing Feynman diagrams for the $g g$-fusion process in second-order perturbation theory. (a) is the $t$-channel diagram, (b) is the $u$-channel-exchange diagram, and (c) is the $s$-channel diagram that includes the threegluon vertex. $\lambda$ and $\lambda^{\prime}$ are the polarizations of the gluons, $\sigma$ and $\sigma^{\prime}$ denote the spins of the outgoing quarks, and, finally, $a$ and $b$ are standing for the gluon color $a, b=1, \ldots, 8$.

a)

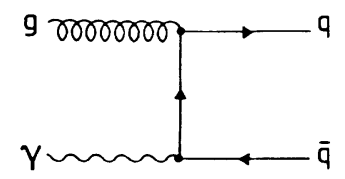

b)

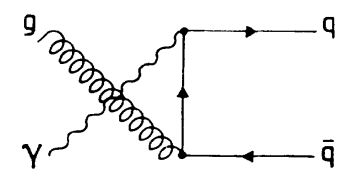

FIG. 8. The $t$-channel diagram and the $u$-channel-exchange diagram of the $\gamma g$-fusion process also representing the secondorder contribution to the $S$ matrix.

$$
\langle f|\mathcal{S}| i\rangle=\delta_{f i}+i(2 \pi)^{4} \delta\left(P_{f}-P_{i}\right)\langle f|M| i\rangle
$$

Hence, we obtain for the annihilation process, using the QCD Feynman rules,

$$
\begin{aligned}
\langle t \bar{t}|\mathcal{M}| q \bar{q}\rangle= & \bar{u}_{\tau}(q) g_{s} \gamma_{v^{\frac{1}{2}}}\left(\lambda^{b}\right)_{k l} v_{\tau^{\prime}}\left(q^{\prime}\right)\left(i \frac{g^{\mu \nu}}{k^{2}} \delta_{a b}\right) \\
& \times \bar{v}_{\sigma^{\prime}}\left(p^{\prime}\right) g_{s} \gamma_{\mu} \frac{1}{2}\left(\lambda^{a}\right)_{i j} u_{\sigma}(p) .
\end{aligned}
$$

This is similar to the well-known $e^{+} e^{-}$-annihilation $M$ matrix element; the electromagnetic coupling constant $e$ is replaced by the strong-coupling constant $g_{s}$. Furthermore, elements $\left(\lambda^{a}\right)_{i j}$ of the generators of color SU(3) appear, which is typical for QCD processes. To determine unpolarized cross sections, one has to square the amplitude, average over initial spins and colors, and sum over final spins and colors. As a consequence, traces of $\lambda$ matrix products occur. These are computed employing the usual Lie-algebra relations. For example, we get

$$
\operatorname{Tr}\left[\lambda^{a} \lambda^{a}\right]=2
$$

Results for these trace operations and some other useful relations are listed in [24]. The unpolarized square of the matrix element becomes

$$
\begin{aligned}
\overline{\left|M_{f i}\right|^{2}}= & \frac{2}{9} \frac{g_{s}^{2}}{s^{2}} \operatorname{Tr}\left[\left(\not p^{\prime}-m_{q}\right) \gamma_{\mu}\left(\not p+m_{q}\right) \gamma_{v}\right] \\
& \times \operatorname{Tr}\left[(\not q+m) \gamma^{\mu}\left(\not q^{\prime}-m\right) \gamma^{\nu}\right] .
\end{aligned}
$$

The determination of the traces and the integration over the phase space is now straightforward, in complete analogy with QED calculations. One is led to Eq. (9).

We have to pay more attention to the $g g$-fusion graph, where we have three coherent contributing Feynman graphs: the $t$-channel process [Fig. 7(a)], the $u$-channelexchange graph [Fig. 7(b)], and the $s$-channel reaction including the three-gluon vertex [Fig. 7(c)]. We write, for the amplitude,

$$
M_{g g}=M_{t}+M_{u}+M_{s}
$$

and, for the square of the amplitude,

$$
\begin{aligned}
\left|M_{g g}\right|^{2}= & \left|\mathcal{M}_{t t}\right|^{2}+\left|\mathcal{M}_{u u}\right|^{2}+\left|\mathcal{M}_{s s}\right|^{2} \\
& +2 \operatorname{Re}\left\{\mathcal{M}_{t} \mathcal{M}_{u}^{*}+\mathcal{M}_{u} \mathcal{M}_{s}^{*}+\mathcal{M}_{s} \mathcal{M}_{t}^{*}\right\} .
\end{aligned}
$$

Explicitly the amplitude is given by 


$$
\begin{aligned}
\mathcal{M}_{g g}=\varepsilon_{\lambda^{\prime}}^{\mu} \varepsilon_{\lambda}^{v} v_{\sigma^{\prime}}\left(q^{\prime}\right) & \left\{\frac{-i}{t-m^{2}} \frac{g_{s}^{2}}{4}\left(\lambda^{a}\right)_{i k}\left(\lambda^{b}\right)_{k j} \gamma_{\mu}\left[k_{t}+m\right] \gamma_{v}+\frac{-i}{u-m^{2}} \frac{g_{s}^{2}}{4}\left(\lambda^{b}\right)_{i k}\left(\lambda^{a}\right)_{k j} \gamma_{v}\left[k_{u}+m\right] \gamma_{\mu}\right. \\
& \left.+\frac{1}{s} \frac{g_{s}^{2}}{2}\left(\lambda^{c}\right)_{i j} f_{c a b} \gamma^{\eta}\left[\left(k_{s}+p\right)_{\mu} g_{\eta v}+(q-p)_{\eta} g_{v \mu}-\left(q+k_{s}\right)_{v} g_{\mu \eta}\right]\right\} \bar{u}_{\sigma}\left(p^{\prime}\right) .
\end{aligned}
$$

We introduce the usual invariant Mandelstam variables $s$, $t$, and $u$. Expressed in these variables, the differential cross section simply reads

$$
\frac{d \bar{\sigma}}{d t}=\frac{1}{16 \pi s^{2}} \overline{\left|\mathcal{M}_{g g}\right|^{2}} \text {. }
$$

In the evaluation of the squared amplitude, one has to deal with the square of the $s$-channel process, but the three-gluon vertex is more difficult to handle since, in the completeness relation,

$$
\begin{aligned}
\sum_{\lambda} \varepsilon_{\mu}(\lambda) \varepsilon_{v}^{*}(\lambda) & \\
= & -\left[g_{\mu v}-\frac{n_{\mu} k_{v}+n_{v} k_{\mu}}{n \cdot k}+\frac{n^{2} k_{\mu} k_{v}}{(n \cdot k)^{2}}\right],
\end{aligned}
$$

the last two terms do not vanish due to current conservation. This is a consequence of the non-Abelian structure of the QCD. One has to insert the full completeness relation. Another way is to use the usual QED polarization sum

$$
\sum_{\lambda} \varepsilon_{\mu}(\lambda) \varepsilon_{v}^{*}(\lambda)=-g_{\mu v}
$$

and to subtract the ghost contribution from the square of the s-channel amplitude. Ghosts are introduced in the QCD Lagrangian to ensure the unitarity of the theory. The amplitude for the $s$-channel process with ingoing ghosts reads

$$
\langle t \bar{t}|M| \zeta \bar{\zeta}\rangle=-i\left[\lambda_{a}, \lambda_{b}\right]_{i j} \frac{g_{s}^{2}}{16} v_{\sigma^{\prime}}(q) p \bar{u}_{\sigma}(p),
$$

where $\xi$ denotes the free ghost. To cancel both unphysical degrees of freedom that are included in Eq. (A10) one has to take into account two different initial ghost states. For both possibilities one yields the same unpolarized, squared amplitude. The total ghost contribution becomes

$$
\overline{\left|M^{G}\right|^{2}}=\frac{\pi^{2} \alpha_{s}^{2}}{s^{2}} \frac{3}{4} \operatorname{Tr}\left\{\left(q^{\prime}-m\right) \not p\left(\not \boldsymbol{p}^{\prime}+m\right)(-\not)\right\} .
$$

The unpolarized, squared amplitudes as a function of the invariant variables are found to be

$$
\overline{\left|\mathcal{M}_{t t}\right|^{2}}=\frac{\pi^{2} \alpha_{s}^{2}}{\left(t-m^{2}\right)^{2}} \frac{8}{3}\left[\left(m^{2}-t\right)\left(m^{2}-u\right)-2 m^{2}\left(m^{2}+t\right)\right],
$$

$\overline{\left|\mathcal{M}_{u u}\right|^{2}}=\frac{\pi^{2} \alpha_{s}^{2}}{\left(u-m^{2}\right)^{2}} \frac{8}{3}\left[\left(m^{2}-u\right)\left(m^{2}-t\right)-2 m^{2}\left(m^{2}+u\right)\right]$,

$$
\overline{\left|M_{s s}\right|^{2}}=\frac{\pi^{2} \alpha_{s}^{2}}{s^{2}} 12\left[2 u m^{2}-u^{2}-s^{2}-u s-s m^{2}-m^{4}\right],
$$

$$
\overline{\left|\mathcal{M}_{t u}\right|^{2}}=-\frac{\pi^{2} \alpha_{s}^{2}}{\left(t-m^{2}\right)\left(u-m^{2}\right)} \frac{2}{3}\left[m^{2}\left(s-4 m^{2}\right)\right] \text {, }
$$

$$
\overline{\left|\mathcal{M}_{u s}\right|^{2}}=\frac{\pi^{2} \alpha_{s}^{2}}{s\left(u-m^{2}\right)} 6\left[2 u m^{2}-s m^{2}-u^{2}-m^{4}\right] \text {, }
$$

$$
\overline{\left|M_{s t}\right|^{2}}=\frac{\pi^{2} \alpha_{s}^{2}}{s\left(t-m^{2}\right)} 6\left[2 u m^{2}+s m^{2}-u^{2}-s^{2}-2 u s-m^{4}\right] \text {, }
$$

$$
\overline{\left|M^{G}\right|^{2}}=\frac{\pi^{2} \alpha_{s}^{2}}{s^{2}} \frac{2}{4}\left[s^{2}-\left(u-m^{2}\right)^{2}-\left(t-m^{2}\right)^{2}\right],
$$

with the abbreviative notation $\overline{\left|\mathcal{M}_{t u}\right|^{2}}=2 \operatorname{Re}\left\{\overline{\mathcal{M}_{t} \mathcal{M}_{u}^{*}}\right\}$, etc. The total cross section is obtained by integration of the differential cross section

$$
\bar{\sigma}_{\text {tot }}(g g \rightarrow t \bar{t})=\frac{1}{16 \pi s^{2}} \int_{t_{\min }}^{t_{\max }} \overline{\left|\mathcal{M}_{g g}\right|^{2}} d t,
$$

where the integration limits are

$$
\begin{aligned}
& t_{\min }=m^{2}-\frac{1}{2} s\left[1+\left(1-\frac{4 m^{2}}{s}\right)^{1 / 2}\right], \\
& t_{\max }=m^{2}-\frac{1}{2} s\left[1-\left(1-\frac{4 m^{2}}{s}\right)^{1 / 2}\right] .
\end{aligned}
$$

Before integrating the differential cross sections one has to eliminate the invariant variable $u$ by the relation

$$
s+t+u=\sum_{i} m_{i}^{2} .
$$

The different contributions to the total cross section are given by

$$
\begin{aligned}
\bar{\sigma}_{t t}(s)=\frac{\pi \alpha_{s}^{2}}{16 s^{2}} \frac{8}{3}[ & -\left(s+4 m^{2}\right) \Delta_{T} \\
& \left.+2\left(m^{2}+\frac{1}{2} s\right) \ln \left\{\frac{1+\Delta_{T}}{1-\Delta_{T}}\right\}\right], \\
\bar{\sigma}_{u u}(s)=\frac{\pi \alpha_{s}^{2}}{16 s^{2}} \frac{8}{3}\left[-\left(s+4 m^{2}\right) \Delta_{T}\right. & \\
& \left.+2\left(m^{2}+\frac{1}{2} s\right) \ln \left\{\frac{1+\Delta_{T}}{1-\Delta_{T}}\right\}\right],
\end{aligned}
$$




$$
\begin{aligned}
& \bar{\sigma}_{s s}(s)=\frac{\pi \alpha_{s}^{2}}{16 s^{2}}\left[-\left(10 s+20 m^{2}\right) \Delta_{T}\right], \\
& \bar{\sigma}_{t u}(s)=\frac{\pi \alpha_{s}^{2}}{16 s^{2}} \frac{4}{3}\left[-\left[m^{2}-\frac{4 m^{4}}{s}\right] \ln \left\{\frac{1+\Delta_{T}}{1-\Delta_{T}}\right\}\right], \\
& \bar{\sigma}_{u s}(s)=\frac{\pi \alpha_{s}^{2}}{16 s^{2}} 6\left[\frac{1}{2} s \Delta_{T}+m^{2} \ln \left\{\frac{1+\Delta_{T}}{1-\Delta_{T}}\right\}\right], \\
& \bar{\sigma}_{s t}(s)=\frac{\pi \alpha_{s}^{2}}{16 s^{2}} 6\left[\frac{1}{2} s \Delta_{T}+m^{2} \ln \left\{\frac{1+\Delta_{T}}{1-\Delta_{T}}\right\}\right],
\end{aligned}
$$

with the threshold function $\Delta_{T}$ defined in Eq. (11). Summation of the terms yields Eq. (12).

The determination of the total $\gamma g$-fusion cross section is now an easy task. One has only to neglect the $s$ channel process and to compute the modified color factors.

\section{APPENDIX B}

The dominant decay channel of the top quark is the weak decay into a $W$ boson and a bottom quark. As long as the top quark is lighter than the sum of the $W$-boson mass and the $b$-quark mass $m_{t}<m_{W}+m_{b}$, it can hardly decay into a real $W$ boson. It was shown that the decay width for such a light top quark has a $m_{t}^{5}$ dependence (see, e.g., [25]). But, since the top quark has to be heavier than $m_{t}>89 \mathrm{GeV}$, the decay into a real $W$ boson is dominant. We want to discuss the calculation for this decay.

From the theory of electroweak interaction it follows that the second-order $M$-matrix element of the top-quark decay process has to be (see Fig. 9 for definitions)

$$
M_{f i}=\Lambda \bar{u}_{r}(q) \gamma^{\mu}\left(1-\gamma_{5}\right) u_{s}(p) \varepsilon_{\mu}^{*}(\sigma)
$$

with

$$
\Lambda=-i \frac{e}{2 \sqrt{2} \sin \vartheta_{W}} V_{t b}^{*} .
$$

For the unpolarized square of the $M$-matrix element we get

$$
\begin{aligned}
\overline{\left|M_{f i}\right|^{2}}= & \frac{1}{2}|\Lambda|^{2}\left(-g_{\mu v}+\frac{q_{\mu}^{\prime} q_{v}^{\prime}}{m_{W}^{2}}\right) \\
\times & \operatorname{Tr}\left\{\left(\not+m_{b}\right) \gamma^{\mu}\left(1-\gamma_{5}\right)\left(\not p+m_{t}\right)\right. \\
& \left.\times \gamma^{\nu}\left(1-\gamma_{5}\right)\right\},
\end{aligned}
$$

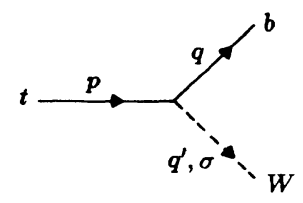

FIG. 9. Diagram for the weak decay of the top quark into a real $W$ boson and a $b$ quark. $p, q$, and $q^{\prime}$ are the four-momenta of the particles and $\sigma$ denotes the $W$-boson polarization. where we inserted

$$
\sum_{\sigma} \varepsilon_{\mu}\left(q^{\prime}, \sigma\right) \varepsilon_{v}^{*}\left(q^{\prime}, \sigma\right)=-g_{\mu v}+\frac{q_{\mu}^{\prime} q_{v}^{\prime}}{m_{W}^{2}}
$$

for the massive vector gauge boson. Evaluation of the trace leads to

$$
\overline{\left|M_{f i}\right|^{2}}=4|\Lambda|^{2}\left[(p q)+\frac{2\left(q^{\prime} q\right)\left(q^{\prime} p\right)}{m_{W}^{2}}\right] .
$$

We write the four-vector products in an explicitly invariant form

$$
\begin{aligned}
& \left(q q^{\prime}\right)=\frac{1}{2}\left(m_{t}^{2}-m_{b}^{2}-m_{W}^{2}\right), \\
& (p q)=\frac{1}{2}\left(m_{t}^{2}+m_{b}^{2}-m_{W}^{2}\right), \\
& \left(p q^{\prime}\right)=\frac{1}{2}\left(m_{t}^{2}-m_{b}^{2}+m_{W}^{2}\right) .
\end{aligned}
$$

Expressed in these terms Eq. (B3) is written as

$$
\overline{\left|M_{f i}\right|^{2}}=2|\Lambda|^{2}\left[m_{t}^{2}+m_{b}^{2}+\frac{\left(m_{t}^{2}-m_{b}^{2}\right)^{2}}{m_{W}^{2}}-2 m_{W}^{2}\right] \text {. }
$$

The differential decay width in the rest system of the top quark is defined by

$$
\begin{aligned}
d \Gamma= & \frac{1}{2 m_{t}}(2 \pi)^{4} \delta^{(4)}\left(p-q-q^{\prime}\right) \\
& \times \frac{d^{3} q}{(2 \pi)^{3} 2 q_{0}} \frac{d^{3} q^{\prime}}{(2 \pi)^{3} 2 q_{0}^{\prime}} \overline{\left|M_{f i}\right|^{2}},
\end{aligned}
$$

with $1 /\left(2 m_{t}\right)$ the invariant flow factor. In the top-quark rest system one obtains

$$
|\mathbf{q}|=\frac{1}{2} m_{t}\left[\lambda\left[1, \frac{m_{b}^{2}}{m_{t}^{2}}, \frac{m_{W}^{2}}{m_{t}^{2}}\right)\right]^{1 / 2}
$$

for the three-momentum of the $b$ quark, where $\lambda(x, y, z)$ is defined in Eq. (19). The integration over $d^{3} q^{\prime}$ is easily evaluated by use of the $\delta$ distribution. Then we transform the integration over $d^{3} q$ into a four-dimensional integral

$$
\begin{aligned}
\frac{d^{3} q}{2 q_{0}} & =\int_{0}^{\infty} d q_{0} \delta\left(q^{2}-m_{b}^{2}\right) d^{3} q \\
& =\int_{-\infty}^{+\infty} d^{4} q \delta\left(q^{2}-m_{b}^{2}\right) \Theta\left(q_{0}\right)
\end{aligned}
$$

The $q_{0}$ integration breaks down due to the remaining energy conservation $\delta$ distribution. The $\delta$ distribution introduced in Eq. (B12) is rewritten as

$$
\begin{aligned}
\delta\left(q^{2}-m_{b}^{2}\right) & =\delta\left(m_{t}^{2}+m_{W}^{2}-m_{b}^{2}-2 m_{t} \sqrt{m_{W}^{2}+|\mathbf{q}|^{2}}\right) \\
& =\left|\frac{q_{0}}{m_{t}^{2} \lambda^{1 / 2}}\right| \delta\left(|\mathbf{q}|-\frac{1}{2} m_{t} \lambda^{1 / 2}\right)
\end{aligned}
$$

The straightforward integration leads to Eq. (18). 
[1] CDF Collaboration, F. Abe et al., Phys. Rev. Lett. 64, 142 (1990).

[2] F. Halzen and D. A. Morris, Part. World 2, 10 (1991).

[3] C. H. Albright, Report No. FERMILAB-CONF-90/196T (unpublished); V. Barger, J. L. Hewett, and T. G. Rizzo, Phys. Rev. Lett. 65, 501 (1990); L. A. Vasilevskaya, A. A. Gvodzdev, and N. V. Mikheev, Pis'ma Zh. Eksp. Teor. Fiz. 51, 443 (1990) [JETP Lett. 51, 501 (1990)].

[4] H. Albrecht et al., Phys. Lett. B 192, 245 (1987); D. P. Roy and S. U. Sankar, ibid. 243, 296 (1990).

[5] P. Abreu et al., Phys. Lett. B 241, 435 (1990); B. Adeva et al., ibid. 237, 136 (1990); S. M. Bilenky, W. Grimus, and H. Neufeld, ibid. 252, 119 (1990).

[6] L. Di Lella, Europhys. News 21, 203 (1990).

[7] P. D. B. Collins and A. D. Martin, Hadron Interactions (Hilger, Bristol, 1984).

[8] H. Sorge, H. Stöcker, and W. Greiner, Ann. Phys. (N.Y.) 192, 266 (1989).

[9] G. Soff, W. Mittelbach, M. Greiner, M. Vidović, Ch. Best, S. Schneider, D. Hilberg, and Ch. Hofmann, in Heavy Ion Physics Today and Tomorrow, Proceedings of the 7th Adriatic International Conference on Nuclear Physics, Brioni, Yugoslavia, 1991, edited by R. Čaplar and W. Greiner (World Scientific, Singapore, 1991), p. 353.

[10] E. Fermi, Z. Phys. 29, 315 (1924); E. J. Williams, Proc. R. Soc. London A139, 163 (1933); C. Weizsäcker, Z. Phys. 88, 612 (1934).

[11] M. Grabiak, B. Müller, W. Greiner, G. Soff, and P. Koch, J. Phys. G 15, L25 (1989).

[12] G. Soff, J. Rau, M. Grabiak, B. Müller, and W. Greiner, in The Nuclear Equation of State, edited by W. Greiner and H. Stöcker (Plenum, New York, 1989), Pt. B, p. 579.
[13] G. Altarelli and G. Parisi, Nucl. Phys. B126, 298 (1977).

[14] D. W. Duke and J. F. Owens, Phys. Rev. D 30, 49 (1984).

[15] Ch. Hofmann, G. Soff, A. Schäfer, and W. Greiner, Phys. Lett. B 262, 210 (1991).

[16] L. Frankfurt and M. Strikman, Phys. Rep. 160, 235 (1988).

[17] M. Greiner, M. Vidović, J. Rau, and G. Soff, J. Phys. G 17, L45 (1991).

[18] M. J. Strassler and M. E. Peskin, Phys. Rev. D 43, 1500 (1991).

[19] J. Feigenbaum, Phys. Rev. D 43, 264 (1991); H. Inazawa, T. Morii, and J. Morishita, in Proceedings of the First Workshop on Japan Linear Collider (JLC), Tsukuba, Japan, 1989, edited by S. Kawabata (KEK Report No. 90-2, Tsukuba, 1990), p. 199; H. Inazawa and T. Morii, Phys. Lett. B 247, 107 (1990).

[20] S. Schneider, H. Sorge, G. Soff, and W. Greiner, J. Phys. G 17, L149 (1991).

[21] H. Baer, V. Barger, J. Ohnemus, and R. J. N. Phillips, Phys. Rev. D 42, 54 (1990).

[22] J. Babcock, D. Sivers, and S. Wolfram, Phys. Rev. D 18, 162 (1978); B. L. Combridge, Nucl. Phys. B151, 429 (1979); M. Glück, J. F. Owens, and E. Reya, Phys. Rev. D 17, 2324 (1978); L. M. Jones and H. W. Wyld, ibid. 17, 1782 (1978).

[23] H. Fritzsch and K.-H. Streng, Phys. Lett. 72B, 385 (1978); M. Glück and E. Reya, ibid. 79B, 453 (1978); L. M. Jones and H. W. Wyld, Phys. Rev. D 17, 759 (1978).

[24] R. D. Field, Applications of Perturbative QCD (AddisonWesley, Redwood City, CA, 1989).

[25] V. D. Barger and R. J. N. Phillips, Collider Physics (Addison-Wesley, Redwood City, CA, 1987); J. H. Kühn and P. M. Zerwas, Phys. Rep. 167, 321 (1988). 